\title{
Transatlantica
}

Revue d'études américaines. American Studies Journal

\section{Abraham Plotkin, un syndicaliste américain observateur à Berlin, 1932-33, du mouvement syndical allemand et de sa chute}

\section{Catherine Collomp}

\section{(2) OpenEdition}

Journals

\section{Édition électronique}

URL : http://journals.openedition.org/transatlantica/254

DOI : $10.4000 /$ transatlantica.254

ISSN : $1765-2766$

Éditeur

AFEA

Référence électronique

Catherine Collomp, «Abraham Plotkin, un syndicaliste américain observateur à Berlin, 1932-33, du mouvement syndical allemand et de sa chute », Transatlantica [En ligne], 1 | 2006, mis en ligne le 05 avril 2006, consulté le 29 avril 2021. URL : http://journals.openedition.org/transatlantica/254 ; DOI : https://doi.org/10.4000/transatlantica.254

Ce document a été généré automatiquement le 29 avril 2021.

\section{(c) (i) $\odot$}

Transatlantica - Revue d'études américaines est mis à disposition selon les termes de la licence Creative Commons Attribution - Pas d'Utilisation Commerciale - Pas de Modification 4.0 International. 


\title{
Abraham Plotkin, un syndicaliste américain observateur à Berlin, 1932-33, du mouvement syndical allemand et de sa chute
}

\author{
Catherine Collomp
}

1 Abraham Plotkin était membre du syndicat de la confection pour Dames, International Ladies Garment Workers' Union (ILGWU), dont il était un militant permanent (organizer) sur la Côte Ouest des Etats Unis ${ }^{1}$. Se trouvant cependant au chômage, comme des millions d'Américains affectés par la grande dépression économique, à l'automne 1932 il décida de partir pour l'Allemagne et d'y passer quelques mois. Ses motivations étaient politiques et syndicales. Plotkin était attiré par la forte tradition socialiste dans le mouvement ouvrier allemand et souhaitait étudier comment celui-ci traitait les questions d'assurance sociale et les problèmes endémiques soulevés par la crise économique. Il avait aussi le pressentiment qu'il allait se passer quelque chose d'important dans ce pays, et comme il le dit dans son journal, «I was tired of reading about things that happened elsewhere ».

2 Il ne croyait pas si bien dire. Arrivé à Berlin en novembre 1932, Plotkin y reste jusqu'en mai 1933. Il est témoin de la prise du pouvoir par Hitler et par le Parti national socialiste, témoin de la destruction complète du mouvement ouvrier allemand ainsi que des premières mesures contre les juifs. Le journal (diary) ainsi que les articles qu'il écrit pendant cette période constituent un témoignage immédiat et direct sur la situation allemande dans ce moment crucial. Fondé sur les entretiens qu'il obtient avec des dirigeants syndicaux et socialistes allemands, le journal de Plotkin rapporte leur interprétation de la crise économique et politique qui allait bientôt les mener au bord du gouffre. La sensibilité américaine de Plotkin, lui aussi syndicaliste et avide d'information politique pour ses compatriotes, transparaît également dans ce récit. Nous nous intéresserons ici principalement à l'échange d'information et de critiques qui s'opère entre syndicalistes allemands et américains. L'analyse à laquelle Plotkin se 
livre sur le mouvement ouvrier allemand offre en effet une comparaison implicite et parfois explicite avec le mouvement américain ne serait-ce que par le choix des questions qu'il pose à ses interlocuteurs et les personnes qu'il rencontre ${ }^{2}$.

Parti des Etats-Unis en octobre 1932, au plus profond de la crise économique, avant même l'élection de Roosevelt et donc avant la mise en place du New Deal, Plotkin pensait trouver dans le mouvement ouvrier allemand un modèle de politique et de pratique sociale dont pourraient s'inspirer les Américains. Ses rencontres avec les principaux dirigeants syndicaux et avec des permanents du Parti social-démocrate allemand (SPD) sont toutes motivées par le souci de s'informer en profondeur sur le modèle de fonctionnement d'un autre type de syndicalisme et de relations sociales. Plotkin, quoique non officiellement mandaté par l'ILGWU, était à cette date l'un des rares syndicalistes américains à sortir de l'isolement géographique et idéologique du mouvement ouvrier de leur pays pour chercher en Europe des solutions à la crise économique. Ce faisant, il raviva également les liens du mouvement ouvrier américain avec ses homologues européens. L'étude de son action et de ses écrits permet de saisir l'un des premiers maillons par lesquels le mouvement américain sortit de son isolement international. Les historiens américains posent en général le moment de la guerre froide comme celui où le mouvement ouvrier américain agit sur la scène internationale. Cet article permet de souligner que ce fut dans les années 1930, dans la lutte contre nazisme et fascisme, que la confédération syndicale américaine, l'American Federation of Labor (AFL), sortit de sa réserve.

4 En Allemagne, Plotkin entre en effet en contact avec les chefs de l'Internationale syndicale, l'International Federation of Trade Unions (IFTU), ou Fédération Syndicale Internationale (FSI), dont le siège était alors à Berlin. En 1919, désapprouvant son orientation socialiste, l'American Federation of Labor avait interrompu ses liens avec la FSI à laquelle elle avait été affiliée en 1913. Elle était donc repliée dans un isolement lié à son apolitisme traditionnel, à sa méfiance à l'égard des mouvements ouvriers européens, mais aussi à l'isolationnisme qui caractérisait les relations internationales américaines depuis la fin de la Première guerre mondiale. En revanche, les fédérations nationales rassemblées dans la FSI étaient fortement engagées dans l'action politique de la gauche non communiste même si leurs rapports avec les partis politiques différaient selon les pays. Dans le cas allemand, le SPD était soutenu politiquement par la forte Confédération Générale des travailleurs allemands (Allgemeiner Deutscher Gewerkschaftsbund, ADGB). C'est dans l'orbite de la Deuxième internationale ouvrière socialiste, Labour and Socialist International (LSI), et non pas de la troisième internationale (communiste), qu'agit la FSI ainsi que le syndicalisme et le Parti social-démocrate allemand. Si l'AFL n'avait pas maintenu son adhésion à la FSI, certains syndicats américains, en revanche, appartenaient toujours aux Secrétariats internationaux (Trade secretariats) qui tentaient d'établir des normes internationales par branches. L'ILGWU, affilié au secrétariat de la confection, était l'un des rares syndicats américains à avoir conservé des liens avec les organisations internationales. Ce lien, ténu et distant, allait être réactivé par l'entremise de Plotkin. Nous verrons que son rôle fut important, tel celui d'un avant-coureur, pour mobiliser l'ILGWU et finalement l'AFL dans le soutien aux victimes du nazisme.

Deux syndicalismes : Condescendance mutuelle.

5 A Berlin, la première personnalité politique que rencontra Plotkin fut Rafael Abramovitch (AP, B4,F2, 23 Nov.32). Cet homme d'une cinquantaine d'années était un 
émigré juif russe, menchevik, ancien bundiste 3 . Réfugié à Berlin depuis la révolution bolchevique, il était membre de la délégation du Parti Ouvrier Social Démocrate Russe en exil, et à ce titre faisait partie du bureau exécutif de l'Internationale socialiste (Labor and Socialist International, LSI). Plotkin l'avait rencontré à Los Angeles, dans les années 1920, alors qu' Abramovitch faisait une tournée de conférences aux Etats-Unis pour soutenir les émigrés mencheviks. Plotkin rendit visite au révolutionnaire russe dont il se sentait proche par conviction politique et parce qu'il était lui aussi un émigré d'origine juive russe. A la différence d'Abramovitch - et plus jeune que lui - il n'avait pas connu la révolution russe, pas même celle de 1905. Les parents de Plotkin étaient parvenus aux Etats-Unis au début du siècle, en 1901, alors qu'il était âgé de 9 ans (AP, B4F1). Malgré la vie américaine de l'un et l'exil allemand de l'autre, Plotkin et Abramovitch appartenaient au même monde juif, laïque et socialiste autrefois organisé par le Bund dans l'Empire russe, et partiellement recomposé en Pologne d'une part, et dans l'exil européen et américain de l'autre. Abramovitch était un correspondant régulier du quotidien yiddish le Jewish Daily Forward et se tenait ainsi informé de l'actualité politique américaine. Plotkin espérait qu'Abramovitch lui faciliterait l'accès au monde politique socialiste et syndical allemand. Leur échange de vues sur les situations américaine et allemande du moment est significatif du fossé entre les cultures syndicales des deux rives de l'Atlantique.

6 D'un côté, Plotkin expliqua qu'il venait pour élucider ce qui faisait la force du syndicalisme allemand malgré le mépris (contempt) en lequel les syndicalistes américains le tenaient. De l'autre, Abramovitch s'enquérant de l'évolution du Parti communiste américain et déplorant les faibles résultats du Parti socialiste aux dernières élections (novembre 1932, 5\%), s'accordait aisément avec Plotkin pour constater l'absence de conscience de classe des ouvriers américains qui ne cherchaient pas de représentation politique.

7 Une plus grande condescendance encore fut marquée à l'égard des syndicats américains par le secrétaire général de la Fédération syndicale internationale (FSI), Walter Schevenels, que Plotkin rencontra en décembre 1933. Schevenels donna une véritable leçon de politique à Abraham Plotkin qui s'informait sur le syndicalisme allemand et européen et fut en quelque sorte accusé de représenter un mouvement infantile :

The only difference between us really is in the point of time and experience. When you have a little more of both, then you will come closer to us, both in your organization forms and in your political views (AP, B4F2, 7 décembre, 1932).

Ce point de vue fut aussi développé par Martin Plettl, secrétaire du syndicat de la confection (Deutscher Bekleidungsarbeiter-Verband, German Needle Trades Alliance) qui trouvait le syndicalisme américain "archaïque et dépassé ", inefficace avec sa centaine d'organisations de métiers qui, par comparaison, ne syndiquaient qu'une fraction des effectifs encadrés par la trentaine de fédérations allemandes, et

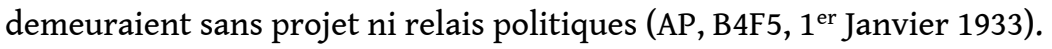

9 L'évident sentiment de supériorité des dirigeants allemands à l'égard du jeune syndicaliste américain et de ce qu'il représentait peut aisément s'expliquer, ne serait-ce que par l'inégale taille des effectifs de part et d'autre, malgré les différences d'échelle, quoique en Allemagne comme aux Etats Unis les mouvements syndicaux aient été durement touchés par la crise. D'une part, la puissante ADGB, qui avait enregistré quelque 8 millions d'adhérents pendant les années 1920, en comptait encore 5 millions 
en 1932. Sur le plan international, aux dires de Schevenels, les effectifs des syndicats allemands représentaient la composante principale des quelque 30 millions d'adhérents de la FSI. Au niveau de la politique intérieure, en outre, l'action syndicale allemande était relayée par le SPD, présent dans les gouvernements de la République de Weimar et de nombreux Länder et dans la direction des municipalités de plusieurs grandes villes. Face à ce puissant mouvement social-démocrate, le syndicalisme américain faisait en effet pâle figure. L'AFL, toujours attachée au syndicalisme de métier, incapable de résister à l'offensive patronale pendant la prospérité des années 1920, avait vu ses effectifs décliner depuis la Première guerre mondiale et plus encore depuis la crise de 1929 et ne comptait guère que 3 millions d'adhérents en 1932 (Bernstein, 1960 et 1970 ; Schevenels).

En Décembre 1932, les propos de Walter Schevenels et des dirigeants allemands pouvaient être justifiés. Mais l'ironie tragique de l'histoire allait donner lieu à un complet renversement de situation. Non seulement le mouvement ouvrier allemand allait se trouver totalement détruit en quelques semaines après la prise de pouvoir nazi, mais ses méthodes elles-mêmes, son légalisme et son réformisme, doivent être mis en question par l'illusion de pouvoir qu'ils lui donnèrent jusqu'à mai 1933. Sans se méprendre sur la nature du nazisme, les dirigeants syndicaux et socialistes allemands se trompèrent certainement sur la capacité de résistance de leurs institutions (Droz; Badia; Braunthal). Inversement, du côté américain, les années 1934-35 et ultérieures allaient donner lieu à un renouveau spectaculaire du point de vue structurel et sur le plan des effectifs qui n'était pas sans rapport avec le modèle allemand. De plus, les réformes sociales préconisées par l'Administration Roosevelt, n'étaient pas loin de s'inspirer de programmes mis en oeuvre par les sociaux-démocrates allemands. Abraham Plotkin se trouvait véritablement à la croisée des chemins.

11 Pendant les premières semaines de son séjour Plotkin fait un tour complet des centrales syndicales allemandes. Il est frappé par l'étendue de leur impact, leurs acquis, y compris en biens matériels, leur organisation et leur structure. Il remarque l'aspect inclusif, intégratif de leur mode d'implantation, c'est-à-dire le syndicalisme d'industrie (industrial unionism par opposition au trade unionism), par branche ou secteur d'activité, encore peu développé outre-Atlantique, mais qui le deviendra avec l'émergence du Congress of Industrial Organizations à partir de 1935. Il en est ainsi non seulement des métallurgistes de la Metallarbeiterverband (regroupant 42 corps de métiers), et des ouvriers de la confection dont Martin Plettl était le président (German Needle Trades alliance), mais aussi des employés municipaux et fonctionnaires tous rassemblés dans le syndicat des Transport and Municipal Workers :

Everyone in Germany who is in any way connected with any governmental department, or industry, from a member of the Reichstag to the women who scrub the floors there ; a doctor in a hospital, a nurse and even the barbers employed there ; transport men, post-office workers, policemen, tramway workers ; every group regardless of its profession, legal, or labor classification, in any way employed by the government, municipal, state or federal, is eligible for membership in the Municipal Workers' Union. This also takes in school teachers, state officials, administrative departments of various types of government departments, university professors-all of them members of the same union (AP, B4F4, 9 décembre 1932).

Emerveillé par cette unité et cette concentration efficace de l'action collective, Plotkin recommande que les divers syndicats des ouvriers du bâtiment aux Etats-Unis, tous 
divisés par métiers, en prennent de la graine (AP, B4, F4, 9 décembre 1932). Venant lui-même du milieu le plus progressiste du syndicalisme américain (la confection), qui ne faisait pas de la qualification ou du métier la base de l'affiliation et organisation syndicale, Plotkin était déjà convaincu du bien-fondé du syndicalisme d'industrie. Mais en Allemagne, l'impact syndical dépassait largement ce que les quelques syndicats d'industrie américains avaient pu réaliser. Il est impressionné par les immeubles modernes que chaque fédération a construits pour établir ses bureaux et développer ses services sociaux et organes de presse. " Among the most beautiful of these buildings are the Metal Workers Building and the Municipal Workers building. They are the modern temples of a modern movement " (AP, B4F5, 27 décembre 1932). Même les ouvriers agricoles, quoique insuffisamment organisés, ont aussi leur syndicat (Deutscher Landsarbeiterverband). Celui-ci proposait sept programmes d'assurances sociales, y compris d'assurance médicale et d'aide à la construction de logements individuels, et avait établi un salaire minimum, des jours fériés, et un maximum horaire au-delà duquel les heures travaillées devaient être payées à 125\% de l'heure normale. Le secrétaire Otto Engel n'eut pas de mal à faire admettre que ces programmes contrastaient avec l'arriération sociale dans laquelle vivaient les ouvriers agricoles dans les états du Sud des Etats-Unis, inféodés à la terre par leur endettement perpétuel en une nouvelle forme de servitude (peonage) (AP, B4F5, 9 décembre 1932). Plotkin apprend aussi que la syndicalisation ne se limite pas catégoriellement au monde ouvrier, mais que les ingénieurs, architectes, dessinateurs et techniciens allemands de différentes professions ont aussi leur syndicat, le Bund der Technischen Angestellten und Beamten, dirigé par Otto Schweizer, affilié à l'ADGB (AP, B4F5, 2 janvier 1933).

La politique sociale des syndicats est un autre sujet d'éducation pour Plotkin. Martin Plettl lui fait visiter les logements sociaux construits depuis une dizaine d'années par des coopératives syndicales dont l'effort était soutenu par des subventions d'Etat. 1,8 million de ces logements avaient été construits depuis 1921 (AP, B4, F5, 3 décembre 1932). Fritz Kummer, rédacteur en chef du Metallarbeiter Zeitung l'informe sur les projets coopératifs, les 52 établissements, les organes de presse et imprimeries, les programmes d'assurance contre les accidents du travail, le chômage et la maladie, et le régime des conventions salariales du syndicat des métallurgistes (AP, B4F5, 27 décembre 1932).

Il est favorablement impressionné par les bourses du travail (Arbeitsamt) dont chaque ville ouvrière et district agricole s'est doté. Elles sont contrôlées de manière tripartite par des représentants des syndicats, des employeurs et des pouvoirs publics (AP, B4F5, 3 janvier 1933). Il assiste à une audience d'un tribunal ayant juridiction sur les conflits du travail, sorte de cours de prud'hommes pour régler les différends sur le lieu de travail. Il s'informe sur la procédure légale pour le licenciement et remarque qu'aux Etats-Unis les ouvriers mis à pied pour fait de grève n'auraient aucune chance de retrouver leur emploi (AP, B4F5, 20 décembre 1932).

15 Au-delà de l'étude des structures syndicales, Plotkin se livre à une véritable enquête sociale dans l'Allemagne pré-hitlérienne. Ses remarques à leur tour céderont la place à l'analyse politique de la situation et à des essais d'interprétation du cataclysme engendré par la prise du pouvoir par le parti nazi. Par des contacts avec des experts du SPD, il étudie l'ampleur du chômage (officiellement 5.109. 000 en octobre 1932), la pauvreté endémique, l'insalubrité de certains quartiers ouvriers qu'il visite - notamment le quartier de Wedding (AP, B4F4, 10 décembre 1932) - , recueille 
des chiffres sur la malnutrition, la prostitution, les problèmes de santé publique (AP, B4F5, 9 et 28 décembre 1932). Il s'informe sur le système d'allocations chômage géré par l'Etat (6 semaines) provenant de contributions des employeurs et employés et comportant une assurance santé. Ces prestations pouvaient être complétées, au besoin, par 14 semaines de secours d'urgence et au-delà de cette période par l'assistance publique dépendant des autorités locales. A la fin de la conversation avec le Dr Haertel, directeur du Berliner Arbeitsamt, Plotkin se voit remettre les formulaires des demandeurs d'emploi, « in the hope that we eventually will have use for them in America ». Ces programmes dépassaient en ampleur ce que le Social Security Act de 1935 allait prévoir trois ans plus tard aux Etats-Unis (AP, B4F7, 15 Janvier 1933). Au coeur du problème économique et social se situait la question du maintien du pouvoir d'achat, qui deviendra la pierre de touche de la politique sociale de Roosevelt, et constituait alors la préoccupation essentielle du SPD. Le Parti social démocrate en effet s'était opposé au gouvernement von Papen qui, en interrompant le régime des négociations collectives, avait fait baisser les salaires. Le SPD préconisait une politique de baisse des prix, d'augmentation des salaires et de programmes de travaux publics destinés à employer 1 million de travailleurs.

Avec George Stolz, secrétaire adjoint de la FSI, et Vatin Hartig, dirigeant du syndicat des employés publics, Plotkin visite aussi un des camps de travail pour la jeunesse (Freiwillige arbeitnehmer camps) qui enrôlaient alors quelque 250000 jeunes gens. Sans salaires, mais pris en charge par l'Etat et les collectivités locales, ces hommes de 16 à 25 ans, logés, nourris, vêtus en uniforme, étaient embauchés pour des travaux de rénovation des parcs, d'entretien des routes et des bâtiments publics. Par sa visite au camp de Plauer, Plotkin comprend que les syndicats, qui s'étaient initialement opposés à ce travail non rémunéré privant de salaires autant de chômeurs qui auraient pu s'en prévaloir, tentent au contraire dorénavant d'en contrôler la gestion afin de les empêcher de tomber entièrement sous la coupe du pouvoir nazi qui les militarisait fortement. D'Amérique, Plotkin en effet avait entendu parler de cette militarisation de la jeunesse. Et l'on ne peut s'empêcher de penser que, d'une part, ces camps préfiguraient le Civilian Conservation Corps qui sera mis en place par le gouvernement Roosevelt en mars 1933, mais de l'autre qu'ils étaient déjà des brigades d'enrôlement de la jeunesse hitlérienne (AP, B4F5, 17 Décembre 1932).

Pour tous les dirigeants syndicaux et SPD que Plotkin rencontre en novembre et décembre 1932, l'accession au pouvoir de Hitler est improbable. «Fascism has not the slightest chance in Germany" affirme Franz Fuchwanger, le responsable du département des statistiques et publications de l'ADGB (AP, B4F2, 29 novembre 1932). Certains pensent qu'une prise de pouvoir communiste est plus dans l'ordre du possible. Ils arguent d'une part du déclin de 2 millions de voix qui ont fait perdre 30 sièges au Reichstag au Parti national socialiste dans les élections du 6 novembre 1932, mais semblent par ailleurs assurés que l'URSS ne pourrait soutenir une révolution communiste en Allemagne même si elle advenait (AP, B4F2, 23 et 29 novembre 1932). Les sociaux-démocrates assistent pourtant impuissants à la radicalisation des masses et surtout des chômeurs, enrôlées soit du côté communiste, soit du côté nazi. Ils n'ont pu enrayer la grève des transports à Berlin en octobre 1932 lancée par les communistes, avec le soutien des nazis, contre la volonté du syndicat et de l'ADGB. Malgré leur superbe - peut-être provoquée par la visite incongrue de l'Américain en ces temps troublés - les dirigeants syndicaux et SPD, sans vouloir l'admettre, voient leur pouvoir miné par la crise et la conjoncture politique. Les attaques des nazis et communistes sur 
leurs effectifs, comme celle du gouvernement von Papen sur leur fonctionnement, ont considérablement affaibli leur assise.

Destruction du mouvement ouvrier allemand

18 La prise de pouvoir par Hitler et les événements qui s'ensuivent donnent rétrospectivement une dimension presque surréaliste au modèle syndical allemand que ses dirigeants décrivent à Plotkin. L'avènement du Troisième Reich, le dernier meeting du SPD, l'incendie du Reichstag, les élections du 5 mars, les assassinats politiques, les violences contre les juifs, la suppression de la presse étrangère et le musellement de la presse libérale interrompent ce récit et le remplacent par des faits que Plotkin ne consigne plus de jour en jour, mais plutôt de sujet en sujet sous forme d'essais. En particulier, dans l'article qu'il envoie à l'American Federationist ${ }^{5}$, Plotkin essaye de comprendre comment le mouvement ouvrier et le Parti social-démocrate, dont il avait admiré les rouages et l'ampleur des programmes, purent si facilement être annihilés. Il décrit l'interdiction complète des publications syndicales et la saisie des imprimeries, la terreur instaurée par les descentes des S.A. dans les sections syndicales locales et centrales dans tout le pays, la saisie des avoirs bancaires et des caisses d'entraide de tous les syndicats, la confiscation et l'occupation de leurs bâtiments, l'arrêt, la torture, l'emprisonnement et l'internement des dirigeants dans des camps de concentration ; il mentionne aussi l'espionnage par infiltration dans les exécutifs syndicaux et l'impossibilité de toute réaction collective. Il décrit également le piège de la manifestation pour la fête du travail du 1er mai, organisée par le pouvoir nazi, à laquelle les organisations syndicales furent contraintes de se rallier, et au lendemain de laquelle une cinquantaine de dirigeants ouvriers furent arrêtés à Berlin et plus encore en dehors de la capitale. Parmi eux figuraient les présidents de l'ADGB, Theodor Leipart et $\mathrm{P}$. Grassmann, et tous les présidents des 31 centrales ouvrières : Martin Plettl de la confection, Hüsemann du syndicat des mineurs, Vormerhaus organisateur du district de Brandebourg, Otto Engel du syndicat des travailleurs agricoles, Otto Schweitzer du syndicat des techniciens, entre autres, tous bien connus de Plotkin. A la suite de ces violences, l'ADGB et les centrales qui lui étaient affiliées, accusées de corruption pour collusion avec le SPD et avec la Fédération Syndicale Internationale, furent placées sous contrôle nazi pour devenir le «Front du Travail». A la fin de son article Plotkin contrecarre toute critique tendant à démontrer l'irresponsabilité ou la naïveté du mouvement syndical allemand :

That their program, because of short-sightedness and over optimism, was defeated, is true. But it should be borne in mind that if they erred in their plans, the criticism hurled at their heads ought not to come from the liberals. If their program was smashed, and the trades unions along with it, it should be remembered that the error in their calculations resulted from the assumption of the left-wingers' policy and not from too much conservatism. ${ }^{6}$

Cette défense répondait aux commentaires visant à suggérer que le SPD aurait dû orchestrer une grève générale en réponse immédiate à la nomination de Hitler au gouvernement, dans les jours suivant le 30 janvier1933. A cette critique Plotkin opposait que les 8 millions de chômeurs (contre 12 millions de salariés employés dans l'industrie) en Allemagne auraient facilement été utilisés pour briser une grève en particulier dans les secteurs clés des transports et des services publics. Il rendait grâce plutôt aux dirigeants ouvriers allemands de s'être sacrifiés pour avoir évité un bain de sang pour la masse des travailleurs. Certes conscient que la division de la gauche entre communistes et socialistes était sa principale source de faiblesse, Plotkin acceptait la 
notion qu'une unité des forces de gauche aurait pu faire échec à l'hitlérisme en ses débuts, mais retenait le point de vue des dirigeants socialistes que l'adhésion des communistes à l'espoir d'une révolution prolétarienne, par la soviétisation des conseils ouvriers dans les usines, et une trop grande inféodation à l'Union soviétique, n'auraient mené qu'à une guerre civile et à une attaque des forces alliées contre l'Allemagne. Plotkin, avait pourtant noté dès février la trop grande assurance des chefs syndicaux qui surestimaient leur force. Son ami Plettl, de la fédération de l'habillement, l'avait assuré de leur immunité :

He did add, that in the event of a real emergency, the machinery of the trade union movement was so geared that every part of Germany could be reached within thirty minutes and every party of the ADGB machine in motion in an hour in every part of the nation. He also intimated that Hindenburg would not tolerate any dictatorship that was established through either sheer terror or through unconstitutional means (AP, B4F6, 10 février 1933).

Réponse américaine

20 A son retour aux Etats-Unis (sans doute précipité7), Plotkin s'employa à faire connaître la persécution contre les dirigeants allemands. Son article sur la destruction du mouvement ouvrier parut en anglais au mois d'août dans l'American Federationist. ${ }^{8}$ Cet article contribua sans doute à convaincre le président de l'AFL, William Green, de lancer un boycott contre les biens et les services allemands. "Apparently (my article) had its effect because our President used it as a basis for the boycott that the Federation passed at its convention last month ", écrivit Plotkin à G.Stolz, maintenant réfugié à Paris (AP, B2F15, 4 novembre 1933). En effet, lors de la convention de l'AFL, Green insista sur le fait que les acquis du mouvement ouvrier allemand, " one of the finest organized trade-union movements ever established by the workers in any country under the sun ", aient pu être balayés en un instant. Il établit un parallèle avec l'AFL, « that we regard as a fixed part of the economic, social and industrial life of our nation ».

And yet, my friends, is there anyone here who can appreciate the fact that overnight this splendid union could be wiped out, its property confiscated, its bank accounts raided and its leaders thrown into prison merely because they belonged to our trade union movement? Such a situation is inconceivable, and yet that is the very thing that happened in Germany. ${ }^{9}$

21 Le président Green, décrivant la destruction du mouvement ouvrier allemand dans les mêmes termes que Plotkin l'avait fait, affirma que le mouvement américain ne pouvait rester silencieux devant une telle attaque: «When those who are bound to us by the bonds of fraternity are persecuted or thrown into prison, when labor organizations are destroyed, I cannot conceive of an American labor movement remaining silent $»^{10}$. Il s'opposa tout aussi fermement à l'antisémitisme : « Do we sit still when the Jewish race is being persecuted in Germany merely because they are Jews? » La proposition d'un boycott des biens et services allemands fut acceptée unanimement, les délégués ayant ovationné l'intervention de leur président. Cette proposition signifiait que l'AFL se ralliait à une décision précédemment prise sur le plan international par la FSI. Aux Etats-Unis c'était l'American Jewish Congress, présidé par le rabbin Stephen Wise, qui avait initialement lancé l'idée du boycott (Gottlieb ; Schevenels, 204-5) ${ }^{11}$.

22 Par ailleurs Plotkin fut aussi actif pour faire venir Martin Plettl aux Etats-Unis. Depuis Paris, Walter Schevenels avait écrit à Plotkin pour solliciter le soutien du mouvement ouvrier américain : «We shall need to have the widest possible international basis, both 
moral and material». Il demandait que soient organisées conférences et collectes de fonds en solidarité avec les victimes allemandes (Schevenels to Plotkin, 3 juin 1933, AP,B2F5 ; Schevenels, 204-5). Martin Plettl fut le premier réfugié allemand à bénéficier de l'aide et du refuge américains. Arrêté, torturé et emprisonné lors des événements de mai 1933, Plettl avait réussi à s'enfuir et à gagner la Hollande où il fut secouru par Van der Heeg, le Président de la Fédération internationale de la confection (dont Plettl était aussi le secrétaire). Il avait alors repris contact avec Plotkin rentré aux Etats-Unis. Celui-ci obtint de David Dubinsky, président de l'ILGWU et de Joseph Schlossberg, secrétaire-trésorier de l'autre grand syndicat de la confection, l'Almagamated Clothing Workers of America (ACWA) d'offrir à Plettl une invitation à faire une tournée de conférences aux Etats-Unis pour informer le monde ouvrier américain des événements allemands. Cette tournée, soutenue également par le Workmen's Circle, association d'entraide juive et laïque, et organisée par le directeur du Jewish Daily Forward, Baruch Charney Vladeck, eut lieu de novembre 1933 à mars 1934. Elle avait pour but d'apporter la vérité sur l'hitlérisme, de développer la propagande antinazie en Allemagne et aux Etats-Unis ainsi que de créer un fonds de solidarité pour les victimes du nazisme dans le mouvement socialiste et syndical allemand ${ }^{12}$. Dans les années ultérieures, Plettl resta aux Etats-Unis, avec sa compagne et ancienne secrétaire Dorothea Heinrich, ils survécurent grâce à l'allocation mensuelle que leur versèrent pendant des années l'ILGWU et l'ACWA, et réussirent finalement à obtenir un visa leur permettant de rester définitivement aux Etats-Unis ${ }^{13}$.

Plotkin lui même, que l'ILGWU avait envoyé organiser les travailleurs de la confection à Kansas City dès son retour, ne fut plus directement actif dans l'organisation du soutien aux victimes du nazisme. Son rôle, s'arrête donc à son retour et l'on ne peut certes pas attribuer le développement de la solidarité américaine à l'égard des mouvements ouvriers européens à sa seule présence à Berlin. Mais les liens qu'il avait créés eurent des prolongements fertiles.

L'ILGWU joua en effet un rôle de premier plan dans l'Internationale syndicale (FSI) dans la lutte contre le nazisme et le fascisme. Ce rapprochement fut occasionné par la reprise de contacts initiés par Plotkin et Plettl qui se traduisirent par la présence, dorénavant régulière, de représentants de ce syndicat dans les congrès de la Fédération internationale de la confection ${ }^{14}$. Mais aussi par l'activité inlassable de David Dubinsky, président de l'ILGWU, qui à partir de 1935, représenta l'AFL au Bureau International du Travail. En 1936 il siégeait aussi au congrès de la FSI à Londres. Au cours des années 1930, Dubinsky fut à l'avant-garde de tous les combats du mouvement syndical américain contre nazisme, fascisme, et antisémitisme . Il joua aussi un rôle central dans la réaffiliation de l'AFL à l'Internationale syndicale en 1937. Le soutien de Dubinsky à la lutte de la FSI pour les républicains espagnols dans la guerre civile, en fut l'occasion ${ }^{15}$.

Le comité de soutien responsable de la venue de Martin Plettl aux Etats-Unis en 1933 préfigurait ce qui allait devenir le Jewish Labor Committee. Il avait été composé des dirigeants de l'ILGWU et de l'ACWA et de Joseph Baskin, président du Workmen's Circle mouvement associatif juif socialiste. Or les mêmes personnes et institutions allaient quelques semaines plus tard fonder le Jewish labor Committee, nouveau mouvement consacré à la défense des victimes du nazisme. Ce fut Baruch Charney Vladeck, émigré juif de la Zone de résidence dans l'empire russe, ancien militant, théoricien et orateur bundiste, devenu aux Etats-Unis directeur général du Jewish Daily Forward, qui donna à 
ce qui avait débuté par le soutien de Martin Plettl l'envergure d'un mouvement entièrement consacré à la lutte contre le fascisme ${ }^{16}$.

Le Jewish Labor Committee (JLC) ${ }^{17}$ se situait à la croisée des mondes juifs, socialistes et syndicaux américains. Son approche théorique et militante de l'antinazisme et de l'antisémitisme était un héritage direct de l'idéologie bundiste de ses fondateurs (Vladeck, Dubinsky Sidney Hillman) qui avaient lutté dans l'Empire russe contre la double oppression tsariste dont les militants ouvriers de la Zone de résidence avaient été l'objet en tant que juifs et en tant que socialistes révolutionnaires. En février 1934, à New York, Vladeck invita les délégués des multiples organisations ouvrières et socialistes juives américaines à s'unir pour former un mouvement de lutte contre la terreur nazie, elle aussi dirigée contre les juifs et l'ensemble du mouvement ouvrier. Cette réunion, qui se produisit quelques semaines après l'arrivée de Plettl aux Etats Unis, amplifiait la solidarité exprimée à son égard. Nombreux furent ensuite les dirigeants syndicaux et socialistes allemands, autrichiens, polonais, italiens qui au long des années 1930, durent au JLC d'être soutenus financièrement, moralement et politiquement. La force logistique et financière de ce mouvement, dont le trésorier était David Dubinsky, reposait sur quelque 500000 adhérents de l'ILGWU, l'ACWA ou du Workmen's Circle entre autres organisations du monde ouvrier juif américain.

L'oeuvre du JLC serait trop longue à détailler ici, mais pour en rendre compte brièvement on peut suggérer que cet organisme, indépendant des centrales syndicales AFL et CIO, mais lié à elles par le biais des syndicats ILGWU et ACWA qui le constituaient, devint l'aile agissante du mouvement ouvrier américain sur le plan international dans les années 1930. Et dans cette entreprise où sont mêlés les réseaux de l'Internationale syndicale FSI comme de l'Internationale socialiste LSI, le JLC sut obtenir le soutient de l'AFL. C'est en son nom par exemple qu'il géra le Labor Chest for the Victims of Nazism and Fascism que l'AFL avait créé en 1934 et qui servit à accueillir et soutenir nombre de réfugiés. L'une des réussites majeures du JLC fut le sauvetage de plusieurs centaines de dirigeants syndicaux et socialistes allemands autrichiens, polonais, mencheviks russes et italiens réfugiés en France, mais pris au piège lors de la chute de ce pays en juin 1940. En contact avec eux depuis 1933-34, le JLC parvint à sauver plusieurs centaines de ces dirigeants en leur fournissant une filière de secours et d'accueil aux Etats-Unis. Parmi ceux-ci figuraient plusieurs des leaders qu'Abraham Plotkin avait rencontrés à Berlin en 1932-33. Ainsi les mencheviks réfugiés dans la région de Toulouse, autour de Rafael Abramovitch, initiateur des contacts de Plotkin à Berlin, furent les premiers à parvenir à New York à l'automne 1940 grâce à l'action du JLC. De même, c'est par ce canal que purent fuir le secrétaire général de l'Internationale socialiste Friedrich Adler, ainsi que Julius Deutsch commandant de la rébellion autrichienne armée en 1934, une centaine de dirigeants du Bund polonais, nombre de militants antifascistes italiens, et du Parti social démocrate allemand et de groupes dissidents socialistes de gauche allemands, ainsi que George Stolz, secrétaire adjoint de l'Internationale syndicale que Plotkin avait connu à Berlin ${ }^{18}$.

En conclusion, on ne saurait mettre en adéquation le rôle d'Abraham Plotkin en 1932-33 et l'opération de sauvetage de la social-démocratie européenne que réalisa en partie le Jewish Labor Committee. Ces indications pourtant permettent de comprendre que les contacts établis par Plotkin furent non seulement prémonitoires, mais se trouvèrent à l'origine d'une chaîne de solidarité qui se développa au cours des années 1930 grâce au JLC et au soutien de l'AFL dont il bénéficia. L'identité juive de Plotkin, ses sympathies 
socialistes sont aussi les caractéristiques qui menèrent le JLC à prendre position dans la lutte antinazie et antifasciste et permirent à cette organisation d'être en contact étroit avec l'appareil syndical et socialiste européen et de sauver un grand nombre d'entre eux, juifs et non juifs, d'une mort quasi certaine.

Plotkin avait assisté malgré lui à l'annihilation du syndicalisme de la République de Weimar et de ses chefs dont il était venu chercher des leçons de politique sociale. Inversement, à son retour aux Etats-Unis en juillet 1933, les premiers effets du New Deal commençaient à favoriser une reprise économique et syndicale. En l'espace de quelques années, le mouvement ouvrier américain allait devenir le plus puissant du monde occidental et le seul à pouvoir alors venir au secours de ses homologues victimes du fascisme. Plotkin avait été témoin de ce retournement de situation tragique du côté européen. Son témoignage, unique dans la littérature syndicale américaine, porte sur un monde dont il avait vu l' anéantissement. Ceci explique peut-être qu'à son retour, plutôt que de publier le recueil de son journal et articles sur l'Allemagne, il se consacra entièrement à la reconstruction syndicale américaine, autre forme de mise en application de ce qu'il avait appris en Europe.

\section{BIBLIOGRAPHIE}

Sources primaires

Abraham Plotkin Collection, Kheel Center of Labor and Management Archives, Cornell University, Ithaca NewYork, Unite, International Ladies Garment Workers' Union (ILGWU) Records.

PLOTKIN, Abraham, "The Destruction of the Labor Movement in Germany », American Federationist, August 1933, 811-826.

Amalgamated Clothing Workers of America, Unite, ACWA Records, Kheel Center, Cornell University, 5619.

American Federation of Labor, Report of Proceedings of the Fifty Third Annual Convention, Washington, D.C., 2-13 october, 1933.

MENDELSUND, Henoch and GOLDWASSER, interviews with Abraham Plotkin in 1978 Unite, ILGWU Records, Oral History, Kheel center.

International Ladies Garment Workers' Union, Report of the 1934 Convention.

International Ladies Garment Workers' Union Records, Unite, Kheel Center, Cornell University, Dubinsky Correspondence.

Isaiah Minkoff Papers, Robert Wagner Labor Archives, Bobst Library, New York University, Jewish Labor Committee Records, Robert Wagner Labor Archives, Bobst Library, New York University.

Justice, organe de l'ILGWU

Sources secondaires 
BADIA, Gilbert, dir. Histoire de l'Allemagne contemporaine, Paris, Messidor Editions sociales, 1987. BERNSTEIN, Irving. The Lean Years, A History of the American Worker, 1920-1933, Boston, Houghton Mifflin, 1960.

The Turbulent Years : A History of the American Worker, 1933-1941, Boston, Houghton Mifflin, 1970.

BRAUNTHAL, Julius. History of the International, vol. I : 1914-43, London, Nelson, 1967.

BREIT, Ernst, ed. Aufstieg des National-sozialismus, Untergang der Republik, Zerschlagung der Gewerkschaften, Cologne, Bund-verlag, 1984.

COLLOMP, Catherine, et GROPPO, Bruno. « Le Jewish Labor Committee et les réfugiés en France, 1940-41 », in Max Lagarrigue, dir., 1940, la France de la défaite, l'Europe du repli, Toulouse, Privat, 2001, 211-47.

COLLOMP, Catherine. «Le Jewish Labor Commitee : Deux générations de réfugiés poliques » in C. Collomp et M. Menéndez, Exilés et réfugiés politiques aux Etat-Unis, Paris, CNRS Editions, 2003, 135-155.

COLLOMP, Catherine. "The Jewish Labor Committee, American Labor, and the Rescue of European Socialists, » International Labor and Working Class History 68 (Fall 2005) : 112-133. DROZ, Jacques. Histoire générale du socialisme, vol. 3, de 1918 à 1945, Paris, PUF, 1977.

FRY, Varian. Surrender on Demand, New York, Random House, 1945, trad. française, La Liste noire, Paris, Plon, 1999

GOTTLIEB, Peter. American Anti-Nazi Resistance, 1933-1941, New York, Ktav Publishing House, 1982.

JACOBS, Jack. « A Friend in Need : The Jewish Labor Committee and Refugees from the German Speaking Lands, 1933-45 », Yivo Annual, 23, 1996, 391-419

LEBOWITZ, Arieh and Gail MALMGREEN. Robert F. Wagner Labor Archives, New York University: The Papers of the Jewish Labor Committee, vol.14 of Archives of the Holocaust, ed. Henry Friedlander and Sybil Milton, Garland, New York, 1993.

LIEBIG, André. From the Other Shore, Russian Social Democracy after 1921, Harvard University Press, 1997.

LIPSTADT, Deborah. Beyond Belief, The American Press and the coming of the Holocaust, 1933-1945, New York Free Press, 1986.

LORWIN, Lewis. The International Labor Movement, History, Policies, Outlook, New York, 1953.

MALMGREEN, Gail. « Labor and the Holocaust, The Jewish Labor Committee and the Anti-Nazi Struggle », Labor's Heritage, 3, Oct. 1991, 20-35.

"Comrades and Kinsmen : The Jewish Labor Committee and Anti-Nazi

Activity », in Christine Collette, and Stephen Bird, eds., Jews, Labour and the Left, 1918-1948, Ashgate, Abingdon, Angleterre, 2000, 4-20.

SCHEVENELS, Walter. Forty Five-Years of the International Federation of Trade Unions, 1901-1945, Bruxelles, 1955.

VAN GOETHEM, Geert. « Conflicting Interests : the International Federation of Trade Unions, (1919-1945) » in Anthony Carew, Michel Dreyfus, Geert van Goethem, et al. The International Confederation of Free Trade Unions, Berne, Peter Lang, 2000, 75-173.

Who's Who in Labor, New York, Dryden Press. 
WINDMULLER, John. American Labor and the International Labor Movement, 1940-53, Ithaca, ILR Press, 1954.

\section{NOTES}

1. La seule référence biographique publiée sur Abraham Plotkin, très succincte, est celle qui apparaît in Who's Who in Labor, New York, Dryden Press, 1946, p. 282. Pour plus d'information voir Henoch Mendelsund and Ethel Goldwasser, Interviews with Abraham Plotkin (Kheel Center for Labor and Management Archives, Cornell University, Unite, ILGWU records, 5780/110 B.15 and 16). Plotkin ,né en 1892 dans la partie ukrainienne de la Zone de résidence de l'Empire russe avait émigré avec ses parents aux Etats-Unis en 1901. Comme beaucoup d'immigrants juifs, il avait très jeune travaillé dans la confection et s'était impliqué dans l'activisme politique du Socialist Party. De 1924 à 1932, il fut organizer pour l'ILGWU à Los Angeles et à San Francisco. Après son retour d'Allemagne en juillet 1933 et pour le reste de sa vie active, il se consacra à nouveau au militantisme syndical.

2. Abraham Plotkin Collection, Kheel Center, ILGWU Records, 6036/016, Cornell University, Ithaca, N.Y. Cité dans cet article comme AP, suivi des références (Boxes and Folders, B,F) et dates. L'édition du journal d'Abraham Plotkin est en préparation : Catherine Collomp and Bruno Groppo, eds. Abraham Plotkin's Diary : An American Labor Leader in Berlin during Hitler's Rise to Power, 1932-33, University of Illinois Press, forthcoming, 2006.

3.Fondé à Vilnius en 1897, le Bund, Union générale des ouvriers juifs de Russie, Lituanie et Pologne, était à la fois un syndicat et un parti politique socialiste. Demandant la reconnaissance de l'identité nationale, non territoriale, juive, il proposait un programme fondé sur la culture laïque de langue yiddish. Le Bund était représenté en tant que tel dans le Parti ouvrier social démocrate russe. Après la révolution russe de 1917 et après la Première guerre mondiale c'est en Pologne que le Bund se recomposa comme parti politique.

4. Voir l'article de Klaus Patel sur ce sujet dans cette livraison de Transtlantica.

5. Abraham Plotkin, «The Destruction of the Labor Movement in Germany ", American Federationist, August 1933, pp.811-826.

6. Ibid, p.825.

7. Fut-il expulsé d'Allemagne ou à court d'argent et désormais sans soutien ? Plotkin se trouve à la mi-mai à Amsterdam hébergé par Van der Heeg, président de la Confédération internationale de l'habillement, d'où il reprend contact avec les syndicalistes de l'ILGWU et les informe de l'arrestation de Plettl notamment, puis il se rend à Paris, avant de retourner aux Etats-Unis, (lettre de S.Lefkovitz à Plotkin, adressée à Amsterdam, 23 mai 1933. AP, B1,F14).

8. Selon Plotkin, l'article fut publié dans le Jewish Daily Forward, en plusieurs livraisons à partir du $1^{\text {er }}$ juillet 1933. Nos recherches grâce à plusieurs lecteurs yiddishophones

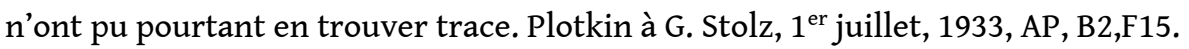
American Federationist,August 1933, pp 811-826.

9. American Federation of Labor, Proceedings of the 1933 Convention, pp.457-471. 10. Ibid. p.468. 
11. La FSI décida d'un boycott contre les produits allemands à son Congrès de juillet 1933 à Bruxelles. Geert van Goethem, « Conflicting Interests, The International Federation of Trade Unions », p. 124.

12. Plotkin to Plettl, July 14, 1933; Nov 1 1933, AP, B2, F9; ILGWU, 1934 Convention, « General Executive Report on Martin Plettl's Tour », p.166. Plett to Joseph Schlossberg, ACWA Records, 5619, B.143; Justice, Nov. 1, 1933, p. 21; Dec. 1933, p. 27.

13. Plettl to Dubinsky and to Schlossberg respectivement in ILGWU et ACWA Records.

14. Isidore Nagler se rendit au congrès de Londres, août 1934; Julius Hochman, General Manager du New York Joint Board, visita les dirigeants en Angleterre, Pologne et Russie dans l'automne 1934, Justice, août 1934, p. 14; jan. 1935, p. 4.

15. AFL, 1937 Convention, pp. 628-30.

16. Né à Dukora en 1886, près de Minsk, dans l'Empire russe, Vladeck était représentant du Bund dans le Parti ouvrier social démocrate russe. Son talent oratoire et sa pensée théorique lui avaient valu le surnom de « deuxième Lassalle » dans les cercles juifs et socialistes. La répression tsariste contre les révolutionnaires le contraignit à l'exil. Il parvint aux Etats-Unis en 1908 et devint correspondant du Jewish daily Forward (1911), puis rédacteur (1916) et enfin directeur (1918-1938) de ce quotidien. Il fut élu au conseil municipal de Brooklyn en tant que socialiste en 1917 et 1919, et de la ville de New York en 1936. Il dirigea plusieurs organismes de secours juifs (People's Relief Committee, HIAS, ORT). Il mourut en 1938. Biographie in Lebowitz and Malmgreen, (1993 p. xxx). 17. Sur le Jewish Labor Committee voir : Malmgreen, ( 1991 et 2000) Lebowitz and Malmgreen (1993) ; Jacobs (1996) ; Collomp et Groppo, (2001) ; Collomp, (2003).

18. La liste des personnes sauvées par le JLC à la date de juillet 1941 se trouve dans les papier de Isaiah Minkoff, organisateur de l'opération de secours du JLC, Isaiah Minkoff Papers, B6.F.10. Sur les opérations de sauvetage voir Jacobs, (1996) ; Collomp et Groppo ( 2001) Collomp (2004).

\section{INDEX}

Keywords : Abraham Plotkin, Jewish Labor Committee, Hitler's rise to power, German Labor Mots-clés : prise de pouvoir par Hitler, mouvement ouvrier allemand

\section{AUTEUR}

\section{CATHERINE COLLOMP}

Université Paris VII 